Supplement of

\title{
Reconciling North Atlantic climate modes: revised monthly indices for the East Atlantic and the Scandinavian patterns beyond the 20th century
}

\section{Laia Comas-Bru and Armand Hernández}

Correspondence to: Armand Hernández (ahernandez@ictja.csic.es)

The copyright of individual parts of the supplement might differ from the CC BY 4.0 License. 
Table S1: Summary of meteorological stations used in this study. Daily datasets were used to calculate monthly series. Grey rows correspond to the selected stations for this study.

\begin{tabular}{|c|c|c|c|c|c|c|c|c|}
\hline Station Name & Coordinates & $\begin{array}{c}\text { Station Data } \\
\text { Availability } \\
\text { Time Period }\end{array}$ & $\begin{array}{l}\text { Selected Time } \\
\text { Interval }\end{array}$ & Resolution & $\begin{array}{c}\text { Monthly } \\
\text { missing data (> } \\
5 \text { days per } \\
\text { month) }\end{array}$ & Measure & Source & $\begin{array}{c}\text { Highest } \\
\text { correlation } \\
\text { with } \\
\text { corresponding } \\
\text { EOFs from all } \\
\text { reanalysis } \\
\text { products } \\
\end{array}$ \\
\hline $\begin{array}{c}3953 \\
\text { VALENTIA }\end{array}$ & $\begin{array}{c}51.93^{\circ} \mathrm{N} 10.25^{\circ} \mathrm{W} \\
14 \text { masl }\end{array}$ & $1866-2002$ & 1866-1938 & Monthly & $\begin{array}{l}05 / 1872 ; \\
05 / 1873 ; \\
05 / 1874 ; \\
12 / 1938\end{array}$ & $\begin{array}{c}\text { Mean sea level } \\
\text { pressure, 0-0 } \\
\text { UTC }\end{array}$ & Met Éireann & \multirow{2}{*}{$\begin{array}{c}0.87 \\
\text { (ERAi) }\end{array}$} \\
\hline $\begin{array}{l}305 / 2275 \\
\text { VALENTIA }\end{array}$ & $\begin{array}{c}51.94^{\circ} \mathrm{N} 10.22^{\circ} \mathrm{W} \\
9 \mathrm{masl}\end{array}$ & 1939-2016 & $1939-2016$ & Daily & ---- & $\begin{array}{c}\text { Sea level } \\
\text { pressure, mean } \\
18-18 \text { UTC } \\
\end{array}$ & ECA\&D & \\
\hline $\begin{array}{c}2230 \\
\text { BERGEN/FLESLAND }\end{array}$ & $\begin{array}{c}60.28^{\circ} \mathrm{N} 05.23 \mathrm{E} \\
48 \mathrm{masl}\end{array}$ & $1957-2017$ & 1957-2017 & Daily & --- & $\begin{array}{c}\text { Sea level } \\
\text { pressure, mean } \\
\text { 18-18 UTC }\end{array}$ & ECA\&D & $\begin{array}{c}0.85 \\
\text { (ERA20C) }\end{array}$ \\
\hline $\begin{array}{c}265 \\
\text { BERGEN/FLORIDA }\end{array}$ & $\begin{array}{c}60.38^{\circ} \mathrm{N} 5.33^{\circ} \mathrm{E} \\
12 \mathrm{~m} \text { asl }\end{array}$ & $1901-2017$ & $1901-2017$ & Daily & --- & $\begin{array}{c}\text { Sea level } \\
\text { pressure, mean } \\
18-18 \text { UTC }\end{array}$ & ECA\&D & $\begin{array}{c}0.87 \\
\text { (ERA20C) }\end{array}$ \\
\hline $\begin{array}{l}1047 \\
\text { SAUDA }\end{array}$ & $\begin{array}{c}59.65^{\circ} \mathrm{N} 06.37^{\circ} \mathrm{E} \\
5 \mathrm{masl}\end{array}$ & $1957-2017$ & 1957-2017 & Daily & $\begin{array}{l}\text { 9/2007; } \\
\text { 9/2016- } \\
03 / 2017 \\
\end{array}$ & $\begin{array}{c}\text { Sea level } \\
\text { pressure, mean } \\
18-18 \text { UTC }\end{array}$ & ECA\&D & $\begin{array}{c}0.85 \\
\text { (ERA20C) }\end{array}$ \\
\hline $\begin{array}{c}2685 \\
\text { SLATTEROY FYR }\end{array}$ & $\begin{array}{l}59.90^{\circ} \mathrm{N} 05.07^{\circ} \mathrm{E} \\
25 \mathrm{masl}\end{array}$ & $1957-2017$ & $1957-2017$ & Daily & $\begin{array}{c}\text { 10/1991; } \\
\text { 10/1999- } \\
\text { 02/2000; 01- } \\
\text { 02/2005; }\end{array}$ & $\begin{array}{l}\text { Sea level } \\
\text { pressure, mean } \\
18-18 \text { UTC }\end{array}$ & ECA\&D & $\begin{array}{c}0.87 \\
\text { (ERA20C) }\end{array}$ \\
\hline $\begin{array}{c}194 \\
\text { UTSIRA FYR }\end{array}$ & $\begin{array}{c}59.30^{\circ} \mathrm{N} 04.88^{\circ} \mathrm{E} \\
55 \text { masl }\end{array}$ & $1957-2017$ & $1957-2017$ & Daily & $03 / 2002$ & $\begin{array}{c}\text { Sea level } \\
\text { pressure, mean } \\
18-18 \text { UTC }\end{array}$ & ECA\&D & $\begin{array}{c}0.87 \\
\text { (ERA20C) }\end{array}$ \\
\hline
\end{tabular}


Table S2: Percentage of SLP variability explained by the corresponding EOF during the given season and for each reanalysis dataset. The "total" column is the variability explained by adding the three main EOFs. EOF1 corresponds always to the NAO. EOF2 and EOF3 correspond to the EA and the SCA, respectively, except in MAM when the EOF3 presents the EA pattern and SCA is not reflected by the three first EOFs (see also Fig. 1 and S1-S4).

\begin{tabular}{|c|c|c|c|c|c|c|c|c|c|c|c|c|c|c|c|c|c|c|c|c|}
\hline & \multicolumn{4}{|c|}{ 20CRv2c } & \multicolumn{4}{|c|}{ ERA-20C } & \multicolumn{4}{|c|}{ ERA-40 } & \multicolumn{4}{|c|}{ ERA-interim } & \multicolumn{4}{|c|}{ NCEP/NCAR } \\
\hline & 苞 & 苂 & 苂 & $\begin{array}{l}\bar{\pi} \\
\stackrel{0}{0}\end{array}$ & 苞 & 苂 & 芒 & $\begin{array}{l}\bar{\pi} \\
\stackrel{0}{\circ} \\
\end{array}$ & 몀 & 苂 & ํㅗㅂ & $\begin{array}{l}\overline{\widetilde{\pi}} \\
\stackrel{0}{\bullet}\end{array}$ & 吉 & 苂 & 䒢 & $\begin{array}{l}\overline{\mathbb{\pi}} \\
\stackrel{0}{\circ}\end{array}$ & 苞 & uㅡㅁ & 芩 & $\begin{array}{l}\bar{\pi} \\
\stackrel{0}{0} \\
\end{array}$ \\
\hline DJF & 39 & 19 & 13 & 71 & 40 & 19 & 13 & 72 & 43 & 17 & 13 & 73 & 46 & 16 & 13 & 75 & 41 & 17 & 13 & 71 \\
\hline MAM & 33 & 13 & 10 & 56 & 37 & 15 & 12 & 64 & 33 & 18 & 14 & 65 & 40 & 16 & 10 & 66 & 34 & 18 & 11 & 63 \\
\hline JJA & 30 & 17 & 14 & 61 & 35 & 11 & 10 & 56 & 36 & 11 & 09 & 56 & 39 & 12 & 10 & 61 & 35 & 10 & 09 & 54 \\
\hline SON & 30 & 17 & 14 & 61 & 28 & 19 & 14 & 61 & 27 & 20 & 13 & 60 & 28 & 21 & 14 & 63 & 26 & 19 & 14 & 59 \\
\hline
\end{tabular}


Table S3:- Correlation coefficients between seasonal $(3 \mathrm{~m})$ EOFs from all the employed reanalysis datasets. Note: all correlations with $p$-val $\leq 0.01$ except $^{\text {(a) }} 0.01<p$-val $\leq 0.05$; ${ }^{\text {(b) }} 0.05<p$-val $\leq 0.1$; and ${ }^{\text {(c) }} p$ val $>0.1$. *EOF2 and EOF3 for JJA have different geographical representation (see main text for further details). ${ }^{* *}$ ERA-interim's EOF2 and EOF3 for JJA have been swapped in order to compare EOFs with the same meaning based on their geographical representation.

\begin{tabular}{|c|c|c|c|c|c|}
\hline EOF1 (DJF) & 20CRv2c & $\begin{array}{l}\text { ERA- } \\
20 C\end{array}$ & $\begin{array}{l}\text { ERA- } \\
40\end{array}$ & $\begin{array}{l}\text { ERA- } \\
\text { interim }\end{array}$ & NCEP/NCAR \\
\hline 20CRv2c & 1 & 0.99 & 0.99 & 0.99 & 0.99 \\
\hline ERA-20C & & 1 & 1 & 0.99 & 1 \\
\hline ERA-40 & & & 1 & 0.98 & 0.99 \\
\hline ERA-interim & & & & 1 & 0.99 \\
\hline NCEP/NCAR & & & & & 1 \\
\hline EOF2 (DJF) & 20CRv2c & $\begin{array}{l}\text { ERA- } \\
20 C\end{array}$ & $\begin{array}{l}\text { ERA- } \\
40\end{array}$ & $\begin{array}{l}\text { ERA- } \\
\text { interim }\end{array}$ & NCEP/NCAR \\
\hline 20CRv2c & 1 & 0.99 & 0.98 & 0.96 & 0.99 \\
\hline ERA-20C & & 1 & 0.99 & 0.97 & 0.99 \\
\hline ERA-40 & & & 1 & 0.96 & 0.99 \\
\hline ERA-interim & & & & 1 & 0.95 \\
\hline NCEP/NCAR & & & & & 1 \\
\hline EOF3 (DJF) & 20CRv2c & $\begin{array}{l}\text { ERA- } \\
20 \mathrm{C}\end{array}$ & $\begin{array}{l}\text { ERA- } \\
40\end{array}$ & $\begin{array}{l}\text { ERA- } \\
\text { interim }\end{array}$ & NCEP/NCAR \\
\hline 20CRv2c & 1 & 0.98 & 0.98 & 0.90 & 0.99 \\
\hline ERA-20C & & 1 & 0.98 & 0.93 & 0.98 \\
\hline ERA-40 & & & 1 & 0.94 & 0.99 \\
\hline ERA-interim & & & & 1 & 0.91 \\
\hline NCEP/NCAR & & & & & 1 \\
\hline
\end{tabular}

\begin{tabular}{|c|c|c|c|c|c|}
\hline $\begin{array}{l}\text { EOF1 } \\
\text { (MAM) }\end{array}$ & 20CRv2c & $\begin{array}{l}\text { ERA- } \\
20 C\end{array}$ & $\begin{array}{l}\text { ERA- } \\
40\end{array}$ & $\begin{array}{l}\text { ERA- } \\
\text { interim }\end{array}$ & NCEP/NCAR \\
\hline 20CRv2c & 1 & 0.96 & 0.98 & 0.99 & 0.97 \\
\hline ERA-20C & & 1 & 0.99 & 0.99 & 0.96 \\
\hline ERA-40 & & & 1 & 1 & 0.97 \\
\hline ERA-interim & & & & 1 & 0.99 \\
\hline NCEP/NCAR & & & & & 1 \\
\hline $\begin{array}{l}\text { EOF2 } \\
\text { (MAM) }\end{array}$ & 20CRv2c & $\begin{array}{l}\text { ERA- } \\
20 C\end{array}$ & $\begin{array}{l}\text { ERA- } \\
40\end{array}$ & $\begin{array}{l}\text { ERA- } \\
\text { interim }\end{array}$ & NCEP/NCAR \\
\hline 20CRv2c & 1 & 0.89 & 0.96 & 0.94 & 0.96 \\
\hline ERA-20C & & 1 & 0.98 & 0.99 & 0.93 \\
\hline ERA-40 & & & 1 & 0.98 & 0.95 \\
\hline ERA-interim & & & & 1 & 0.95 \\
\hline NCEP/NCAR & & & & & 1 \\
\hline $\begin{array}{l}\text { EOF3 } \\
\text { (MAM) }\end{array}$ & 20CRv2c & $\begin{array}{l}\text { ERA- } \\
20 C\end{array}$ & $\begin{array}{l}\text { ERA- } \\
40\end{array}$ & $\begin{array}{l}\text { ERA- } \\
\text { interim }\end{array}$ & NCEP/NCAR \\
\hline 20CRv2c & 1 & 0.92 & 0.96 & 0.83 & 0.97 \\
\hline ERA-20C & & 1 & 0.96 & 0.88 & 0.96 \\
\hline ERA-40 & & & 1 & 0.95 & 0.99 \\
\hline ERA-interim & & & & 1 & 0.89 \\
\hline NCEP/NCAR & & & & & 1 \\
\hline
\end{tabular}

\begin{tabular}{|c|c|c|c|c|c|}
\hline EOF1 (JJA) & 20CRv2c & $\begin{array}{l}\text { ERA- } \\
20 C\end{array}$ & $\begin{array}{l}\text { ERA- } \\
40\end{array}$ & $\begin{array}{l}\text { ERA- } \\
\text { interim }\end{array}$ & NCEP/NCAR \\
\hline 20CRv2c & 1 & 0.91 & 0.97 & 0.98 & 0.97 \\
\hline ERA-20C & & 1 & 0.97 & 0.97 & 0.96 \\
\hline ERA-40 & & & 1 & 0.99 & 0.99 \\
\hline ERA-interim & & & & 1 & 0.98 \\
\hline NCEP/NCAR & & & & & 1 \\
\hline *EOF2 (JJA) & 20CRv2c & $\begin{array}{l}\text { ERA- } \\
20 C\end{array}$ & $\begin{array}{l}\text { ERA- } \\
40\end{array}$ & $\begin{array}{l}* * \text { ERA- } \\
\text { interim }\end{array}$ & NCEP/NCAR \\
\hline 20CRv2c & 1 & 0.85 & 0.90 & 0.87 & 0.92 \\
\hline ERA-20C & & 1 & 0.89 & 0.84 & 0.87 \\
\hline ERA-40 & & & 1 & 0.89 & 0.92 \\
\hline $\begin{array}{l}* * \text { ERA- } \\
\text { interim }\end{array}$ & & & & 1 & 0.94 \\
\hline NCEP/NCAR & & & & & 1 \\
\hline *EOF3 (JJA) & 20CRv2c & $\begin{array}{l}\text { ERA- } \\
20 C\end{array}$ & $\begin{array}{l}\text { ERA- } \\
40\end{array}$ & $\begin{array}{l}* * \text { ERA- } \\
\text { interim }\end{array}$ & NCEP/NCAR \\
\hline 20CRv2c & 1 & 0.60 & 0.76 & 0.69 & 0.45 \\
\hline ERA-20C & & 1 & $0.18 a$ & 0.94 & 0.87 \\
\hline ERA-40 & & & 1 & $0.25 a$ & $0.05 b$ \\
\hline $\begin{array}{l}* * \text { ERA- } \\
\text { interim }\end{array}$ & & & & 1 & 0.94 \\
\hline NCEP/NCAR & & & & & 1 \\
\hline
\end{tabular}

\begin{tabular}{|c|c|c|c|c|c|}
\hline EOF1 (SON) & 20CRv2c & $\begin{array}{l}\text { ERA- } \\
20 C\end{array}$ & $\begin{array}{l}\text { ERA- } \\
40\end{array}$ & $\begin{array}{l}\text { ERA- } \\
\text { interim }\end{array}$ & NCEP/NCAR \\
\hline 20CRv2c & 1 & 0.91 & 0.96 & 0.93 & 0.92 \\
\hline ERA-20C & & 1 & 0.97 & 0.95 & 0.93 \\
\hline ERA-40 & & & 1 & 0.99 & 0.99 \\
\hline ERA-interim & & & & 1 & 0.99 \\
\hline NCEP/NCAR & & & & & 1 \\
\hline EOF2 (SON) & 20CRv2c & $\begin{array}{l}\text { ERA- } \\
20 \mathrm{C}\end{array}$ & $\begin{array}{l}\text { ERA- } \\
40\end{array}$ & $\begin{array}{l}\text { ERA- } \\
\text { interim }\end{array}$ & NCEP/NCAR \\
\hline 20CRv2c & 1 & 0.98 & 0.95 & 0.92 & 0.89 \\
\hline ERA-20C & & 1 & 0.98 & 0.96 & 0.94 \\
\hline ERA-40 & & & 1 & 0.97 & 0.98 \\
\hline ERA-interim & & & & 1 & 0.97 \\
\hline NCEP/NCAR & & & & & 1 \\
\hline EOF3 (SON) & 20CRv2c & $\begin{array}{l}\text { ERA- } \\
20 C\end{array}$ & $\begin{array}{l}\text { ERA- } \\
40\end{array}$ & $\begin{array}{l}\text { ERA- } \\
\text { interim }\end{array}$ & NCEP/NCAR \\
\hline 20CRv2c & 1 & 0.96 & 0.98 & 0.94 & 0.97 \\
\hline ERA-20C & & 1 & 0.93 & 0.89 & 0.91 \\
\hline ERA-40 & & & 1 & 0.93 & 0.98 \\
\hline ERA-interim & & & & 1 & 0.95 \\
\hline NCEP/NCAR & & & & & 1 \\
\hline
\end{tabular}



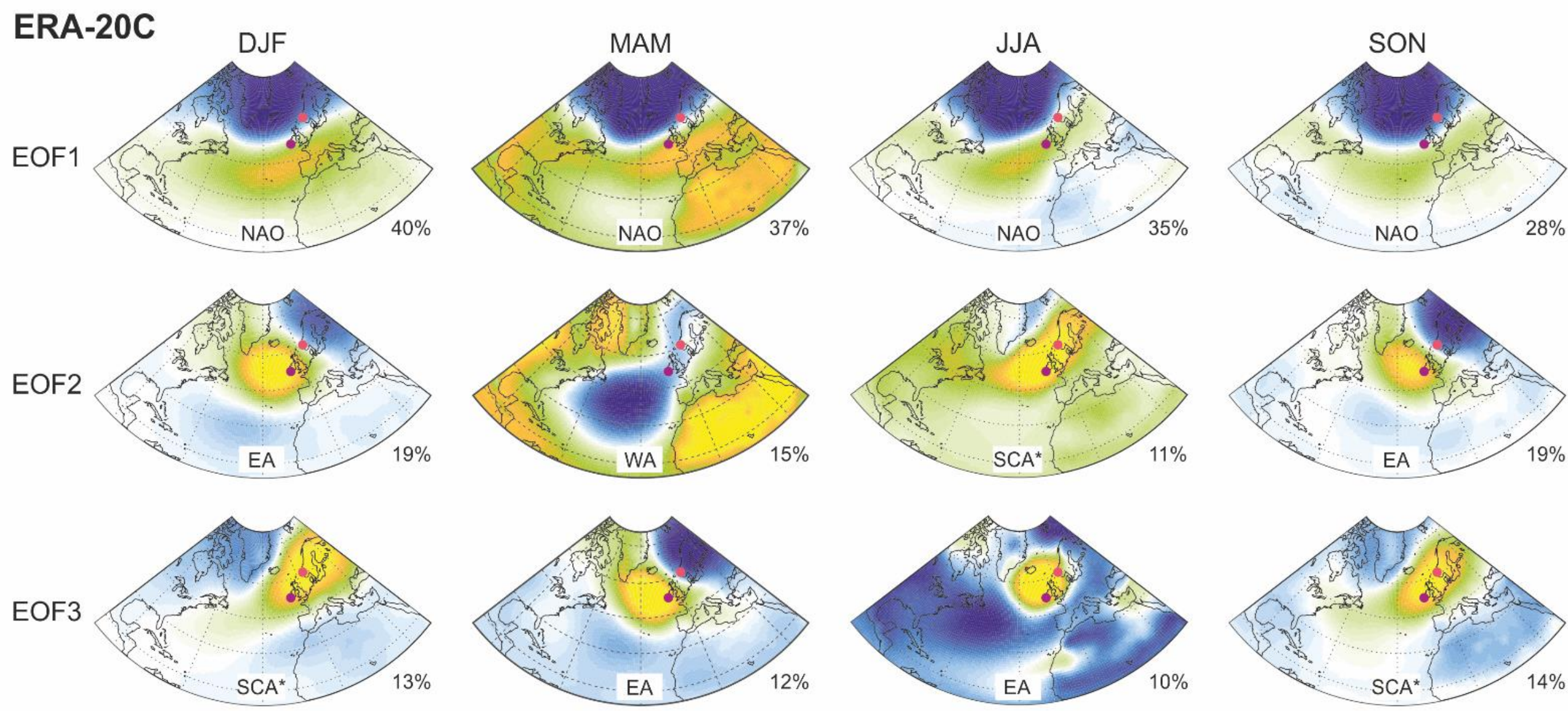

$-8$

$-6$

$-4$

$-2$

0

2

4

6

8

Figure S1: EOF loadings based on monthly SLP data (ERA-20C dataset; Poli et al., 2016). Each column represents a 3-month season. The percentages at the bottom right of each map are the variability explained by the corresponding EOF (rows) at any given season (columns) as shown in Table S2. The text at the bottom of each map identifies the observed pattern. Pink (purple) dots show the location of Bergen Florida (Valentia Observatory) stations as listed in Table 1.

Figures S1-S4 show the same maps for the other four reanalysis products in Table 2. 


\section{ERA-40}
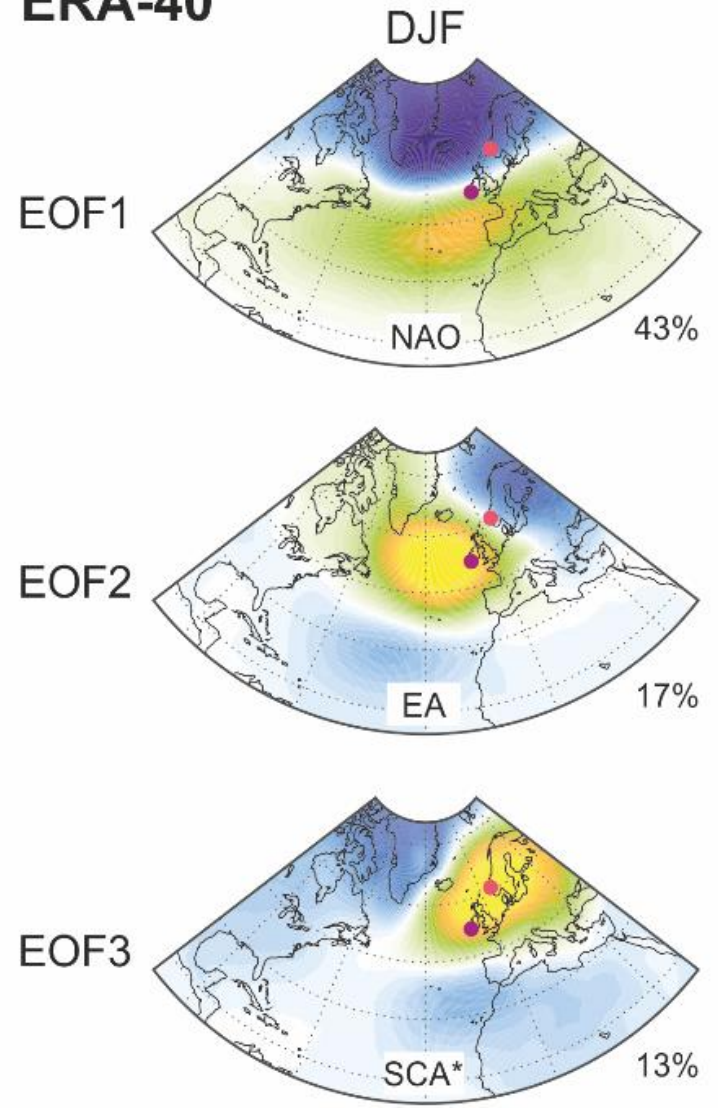
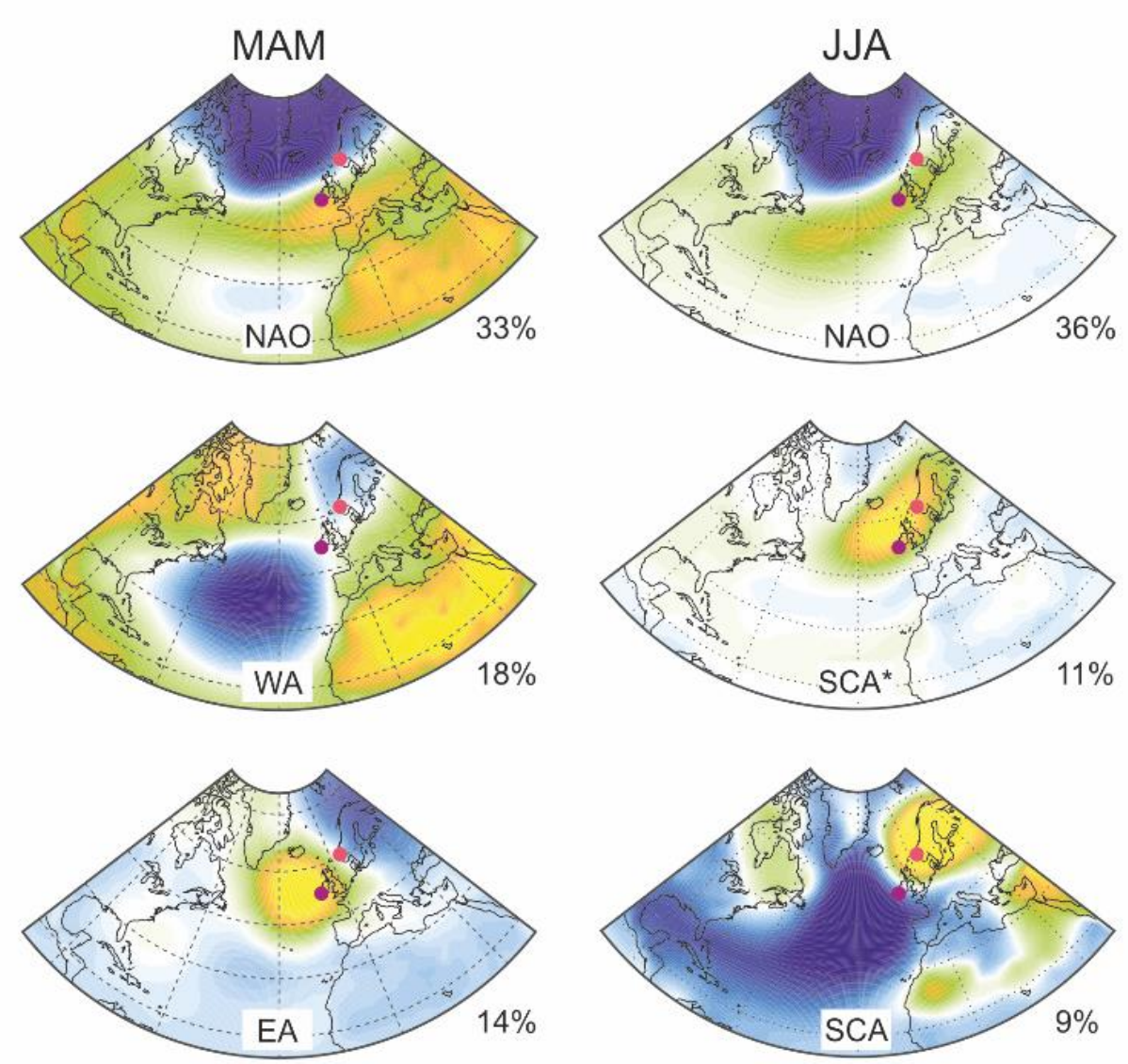
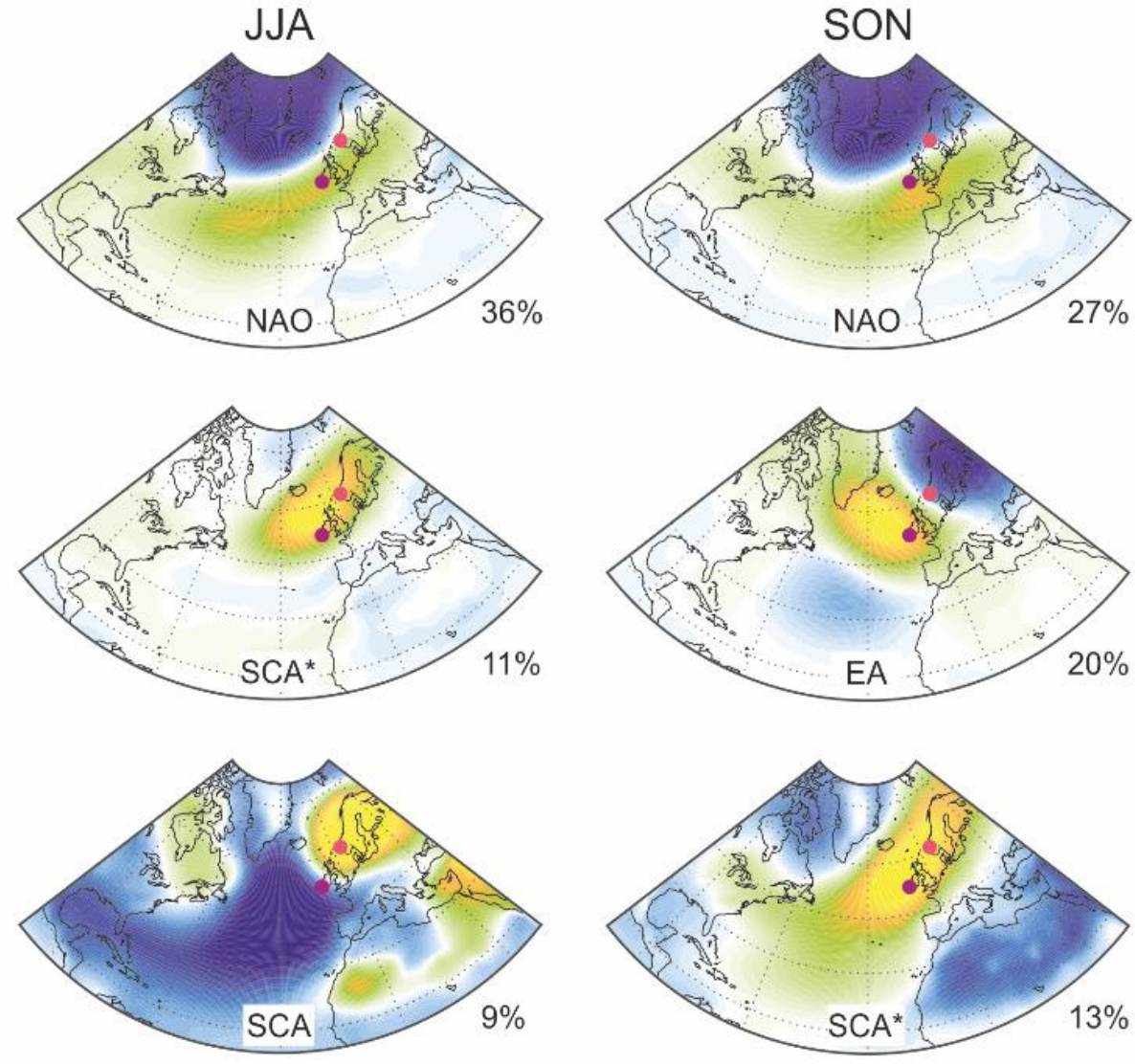

$-8$

$-6$

$-4$

$-2$

0

2

4

6

8

Figure S2: EOF loadings based on monthly SLP data (ERA-40 dataset; Uppala et al., 2005). Each column represents a 3-month season. The percentages at the bottom right of each map are the variability explained by the corresponding EOF (rows) at any given season (columns) as shown in Table S2. The text at the bottom of each map identifies the observed pattern. Pink (purple) dots show the location of Bergen Florida (Valentia Observatory) stations as listed in Table 1.

Figures S1-S4 show the same maps for the other four reanalysis products in Table 2. 

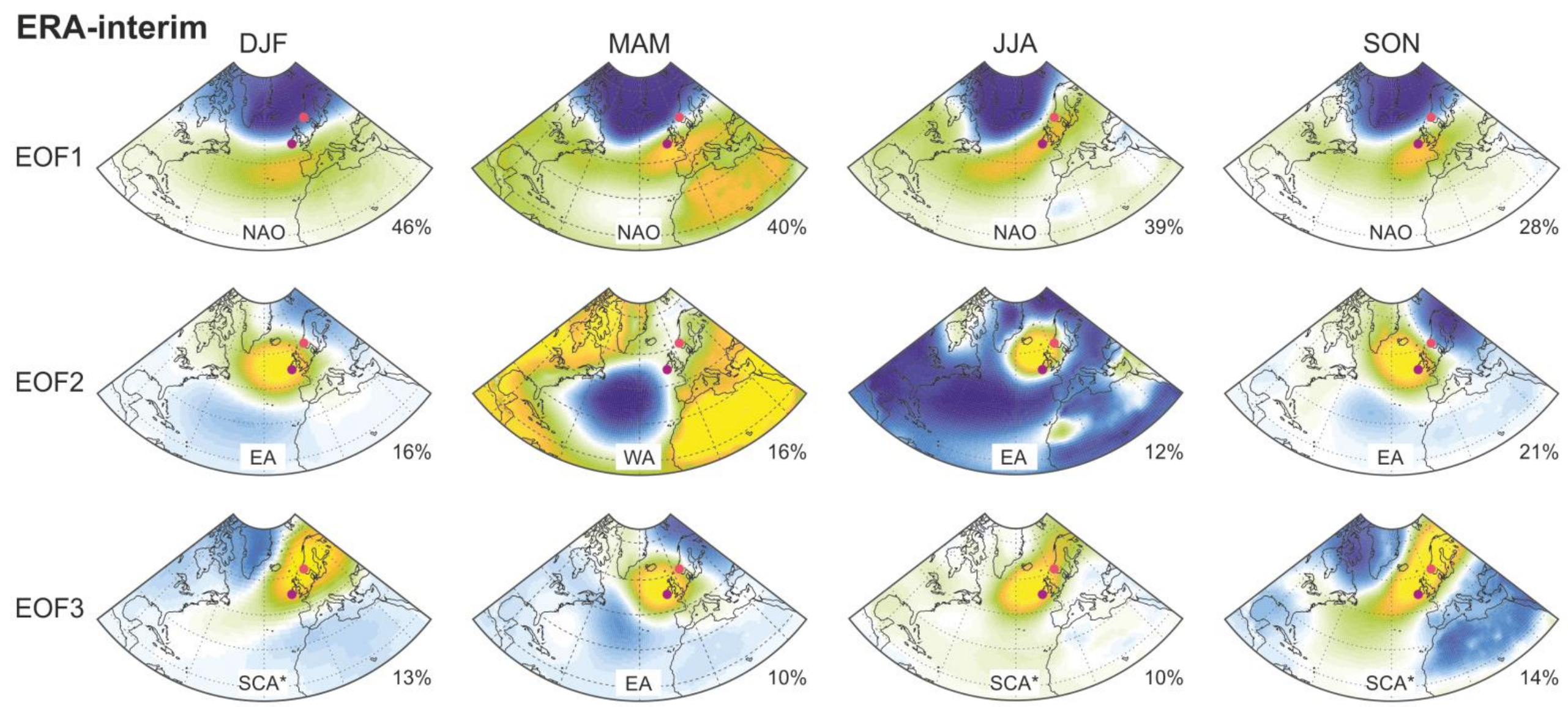

$-8$

$-6$

$-4$

$-2$

0

2

4

6

8

Figure S3: EOF loadings based on monthly SLP data (ERA-interim dataset; Dee et al., 2011). Each column represents a 3-month season. The percentages at the bottom right of each map are the variability explained by the corresponding EOF (rows) at any given season (columns) as shown in Table S2. The text at the bottom of each map identifies the observed pattern. Pink (purple) dots show the location of Bergen Florida (Valentia Observatory) stations as listed in Table 1.

Figures S1-S4 show the same maps for the other four reanalysis products in Table 2. 
NCEP/NCAR
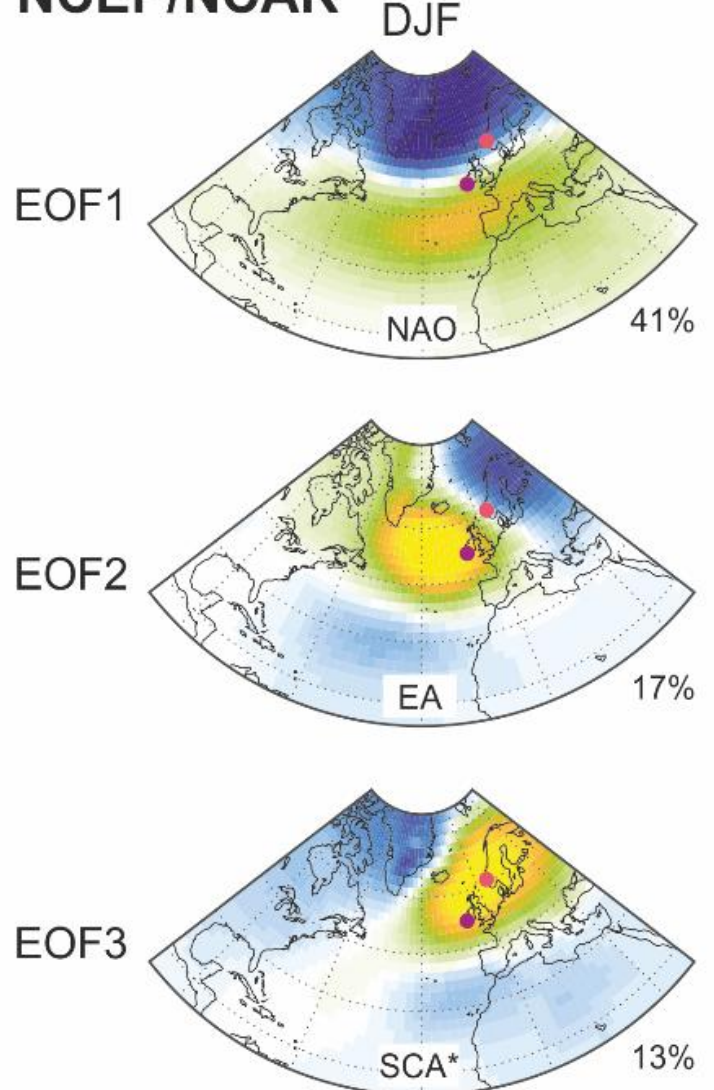
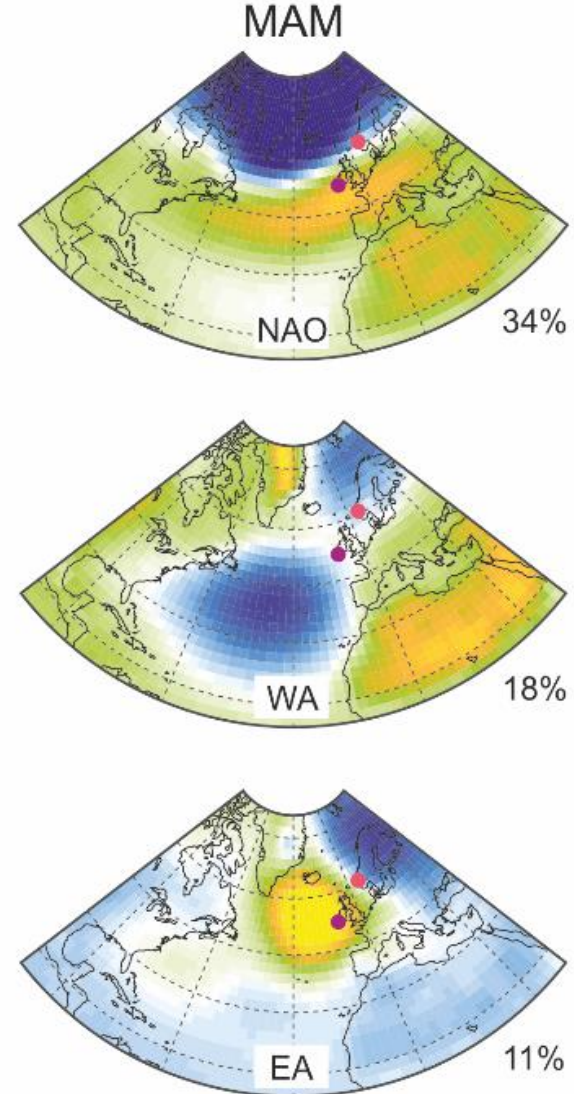
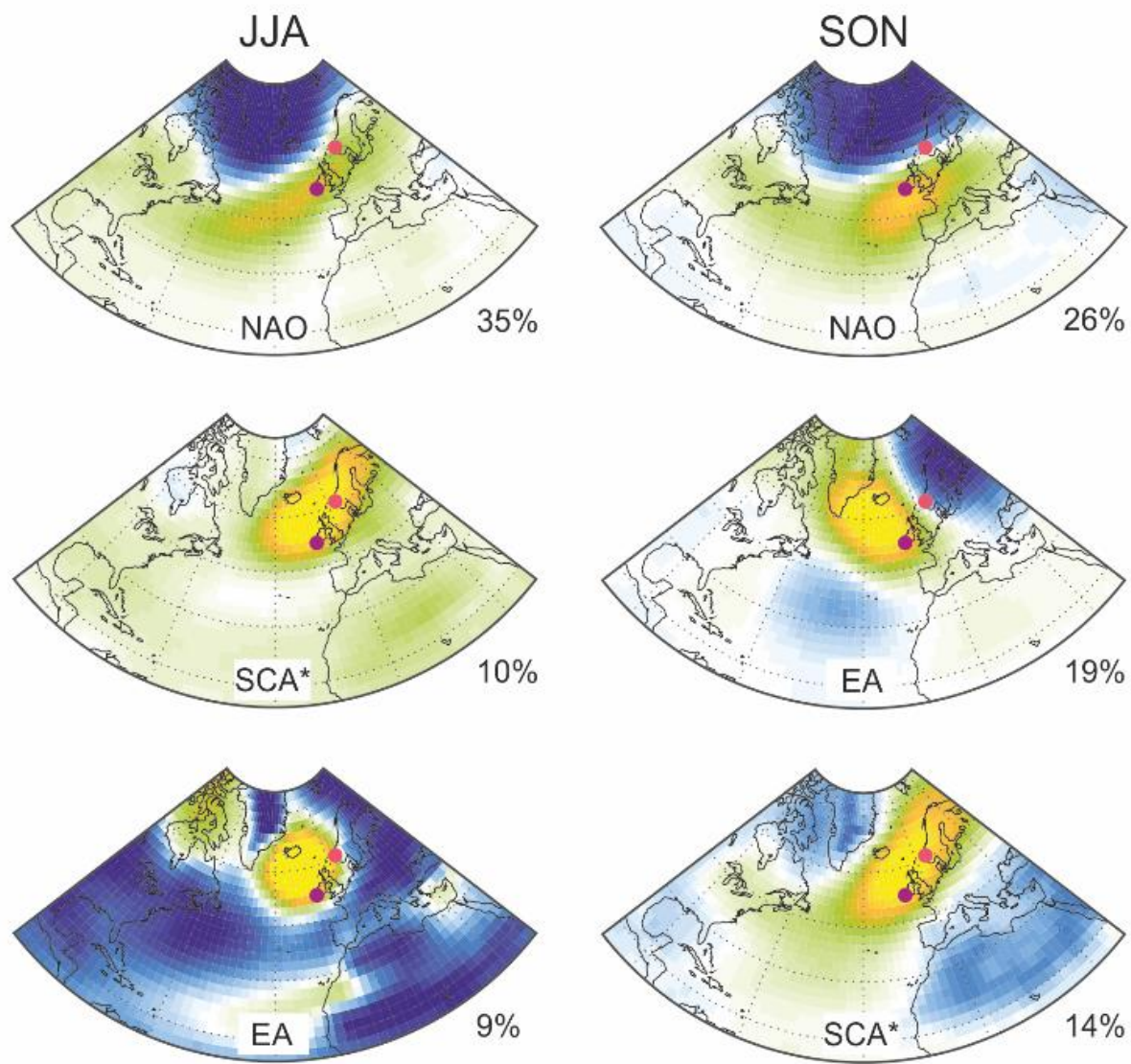

$-8$

$-6$

$-4$

$-2$

0

2

Figure S4: EOF loadings based on monthly SLP data (NCEP/NCAR dataset; Kalnay et al., 1996). Each column represents a 3-month season. The percentages at the bottom right of each map are the variability explained by the corresponding EOF (rows) at any given season (columns) as shown in Table S2. The text at the bottom of each map identifies the observed pattern. Pink (purple) dots show the location of Bergen Florida (Valentia Observatory) stations as listed in Table 1. Figures S1-S4 show the same maps for the other four reanalysis products in Table 2. 